\title{
Selective gas sensors from flames for breath analysis
}

\author{
Andreas T. Güntner, Sotiris E. Pratsinis \\ Particle Technology Laboratory, ETH Zürich, Sonneggstr. 3, 8092 Zürich, Switzerland \\ Corresponding author's e-mail address: Andreas.Guentner@ptl.mavt.ethz.ch
}

\begin{abstract}
Breath analysis is a challenging task for chemical sensors as single tracers need to be detected selectively down to low parts-per-billion (ppb) concentrations. Here, I will present recent achievements of flame-aerosol technology on selective sensing material design and showcase a breath study with Si-doped $\mathrm{WO}_{3}$ acetone sensors for fat burn monitoring. In specific, this sensor was tested on 20 volunteers during exercise and rest to measure their individual breath acetone concentrations in good agreement to proton transfer reaction time-of-flight mass spectrometry (PTR-TOF-MS). During exercise, this sensor revealed clearly the onset and progression of increasing breath acetone levels that indicate intensified fat metabolism, as validated by venous blood $\beta$-hydroxybutyrate (BOHB) measurements. As a result, this simple breath acetone sensor enables easy and hand-held fat burn monitoring for personalized and immediate feed-back on workout that can guide dieting as well.
\end{abstract}

Key words: Metal oxide, acetone, fat burn, flame spray pyrolysis, breath sampling

\section{Introduction}

Flame aerosol technology is an asset in exploring new sensing materials with unprecedented performance due to its wide material flexibility and close control over morphology and composition at the nanoscale. High sensitivity and selectivity are found in metastable crystal phases, solid solutions and mixed oxides, often not accessible by wetphase methods. This has led recently to Sidoped $\mathrm{MoO}_{3}$ and Ti-doped $\mathrm{ZnO}$ for ammonia-[1] and isoprene-selective[2] sensing, respectively.

Even sensor arrays with distinct compositions are possible to overcome selectivity limitations of single materials (e.g. formaldehyde[3]) while nearly orthogonal arrays facilitate the accurate multi-tracer detection for search and rescue application[4]. Further selectivity enhancements are achieved by ad-/absorption[5] or sizeselective (microporous) [6] filters.

In this presentation, I will give a brief overview over selective material design with flame spray pyrolysis and highlight a breath study on 20 volunteers with Si-doped $\mathrm{WO}_{3}$ acetone sensors to monitor individual fat burn rates during exercise and rest.[7]

\section{Experimental}

Si-doped (10 mol \%) $\varepsilon-\mathrm{WO}_{3}$ nanoparticles were prepared by flame spray pyrolysis (FSP) and directly deposited onto $\mathrm{Al}_{2} \mathrm{O}_{3}$ substrates with interdigitated electrodes. By operating them at $350{ }^{\circ} \mathrm{C}$, optimal acetone sensitivity and selectivity was achieved. These sensors were combined with a tailor-made breath sampler for standardized, monitored $\left(\mathrm{CO}_{2}\right.$, airway pressure and flow) and buffered end-tidal breath extraction. Results were validated by PTR-TOFMS and venous blood assay. This study was approved by the local ethics commission.

\section{Results \& Discussions}

The sampler-sensor system was applied to monitor the breath acetone dynamics of 20 volunteers during exercise and postexercise rest. Therefore, all subjects underwent exercise with initial three times 30 min cycling on an ergometer at moderate intensity to stimulate their body fat metabolism followed by a $3 \mathrm{~h}$ rest. (Fig. 1a,b). In a typical case (e.g., subject \#18, red diamonds), breath acetone increases only little during exercise but triples during the postexercise rest (Fig. 1c). This should reflect enhanced body fat metabolism, with acetone being a byproduct of lipolysis. Thus, for subject $\# 18$, it seems that the initial exercise stimulated body fat metabolism that becomes most pronounced after exercise. To confirm this, venous blood BOHB is analyzed simultaneously, as a marker for body fat metabolism (Fig. 1d). Remarkably, it shows the 

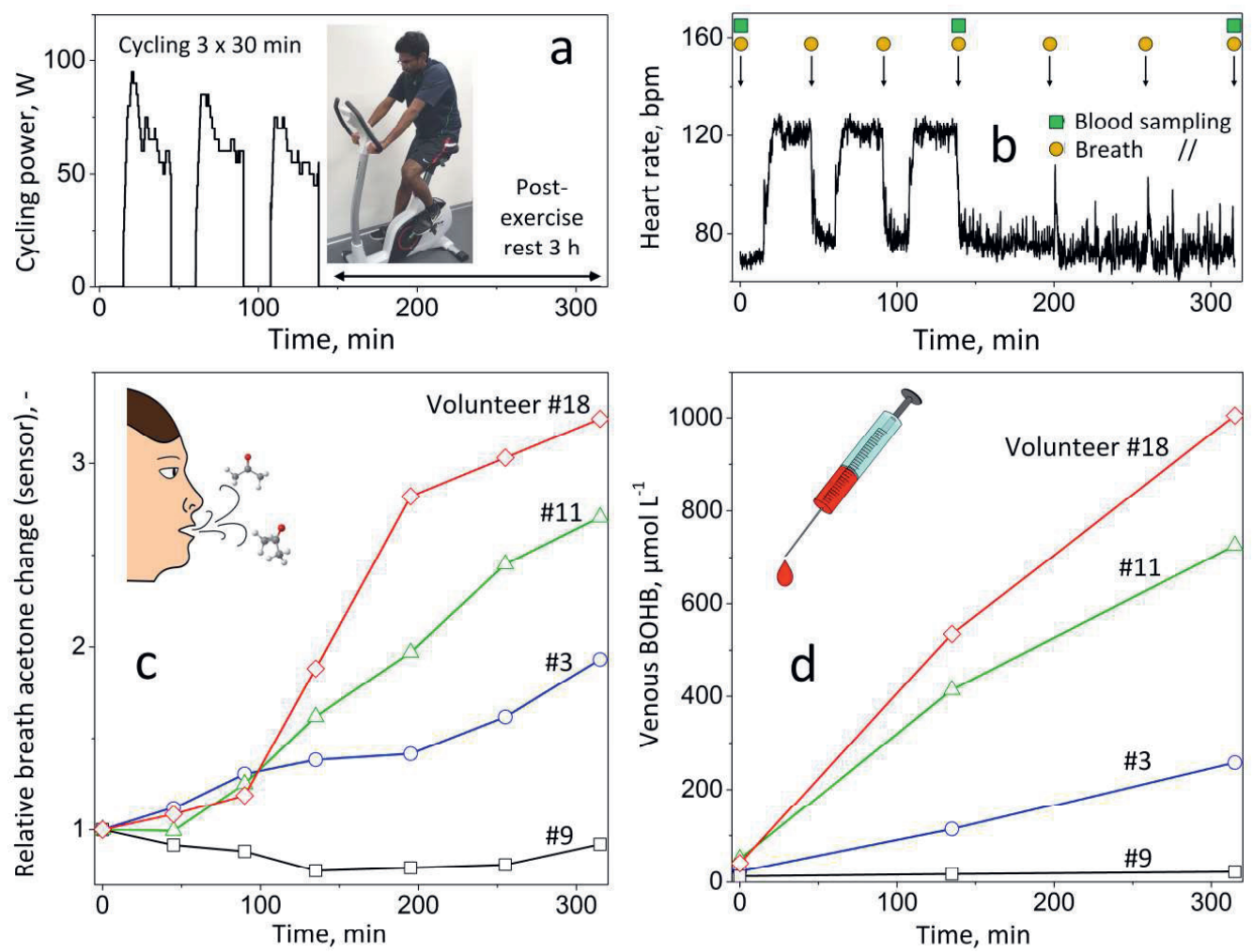

Fig.1. Body fat burn monitoring during exercise and rest. Typical cycling power profiles (a) and heart frequency (b) of a subject when undergoing a testing course with $3 \times 30$ min cycling on an ergometer and $3 \mathrm{~h}$ post-exercise rest. Symbols in (b) indicate breath (orange circles) and venous blood (green squares) sampling. (c) Individual breath acetone changes (relative to initial value) measured by a flame-made Si-doped $\mathrm{WO}_{3}$ sensor of representative subjects during the testing course. (d) Corresponding venous BOHB concentrations that were sampled only 3 times instead of 7 as with breath (b) to minimize volunteer's discomfort. Adopted from [7].

same dynamic response, supporting that the present breath acetone sensor indeed follows body fat metabolism but, most importantly, in a noninvasive manner.

Individual body fat burn rates may differ between humans due to the usual biological variability (including different fitness levels). Nevertheless, the sensor should recognize this correctly for customized feedback. In fact, when comparing the individual exercise profiles of selected volunteers (Fig. 1c) distinctly different breath acetone profiles are detected, again all in good agreement to BOHB dynamics (Fig. 1d).

Finally, to cross-validate the sensor's accuracy for breath acetone detection, all breath samples were analyzed simultaneously by PTR-TOFMS. Both instruments correlate strongly (Pearson's correlation coefficient 0.97, p < 0.05 ) for all 280 samples. This is remarkable considering the sensors simple design, compactness and low cost compared to PTRTOF-MS.

As a result, a portable, easy-in-use and inexpensive breath acetone sensor is presented that is promising for daily application at home or in gyms to provide immediate feed-back during exercise and dieting for more effective body fat loss.

\section{References}

[1] A.T. Güntner, M. Righettoni, S.E. Pratsinis, Selective sensing of $\mathrm{NH}_{3}$ by Si-doped $\alpha-\mathrm{MoO}_{3}$ for breath analysis, Sens. Actuators B, 223 (2016) 266-273. doi: 10.1016/j.snb.2015.09.094

[2] A.T. Güntner et al., Selective sensing of isoprene by Ti-doped $\mathrm{ZnO}$ for breath diagnostics, J. Mater. Chem. B, 4 (2016) 5358-5366. doi: 10.1039/C6TB01335J

[3] A.T. Güntner et al., E-Nose sensing of low-ppb formaldehyde in gas mixtures at high relative humidity for breath screening of lung cancer?, ACS Sens., 1 (2016) 528-535. doi: 10.1021 /acssensors.6b00008

[4] A.T. Güntner et al., Sniffing Entrapped Humans with Sensor Arrays, Anal. Chem., (2018). doi: 10.1021/acs.analchem.8b00237

[5] J. van den Broek, A.T. Güntner, S.E. Pratsinis, Highly Selective and Rapid Breath Isoprene Sensing Enabled by Activated Alumina Filter, ACS Sens., 3 (2018) 677-683. doi: 10.1021/acssensors.7b00976

[6] A.T. Güntner et al., Zeolite membranes for highly selective formaldehyde sensors, Sens. Actuators B, 257 (2018) 916-923. doi: 10.1016/j.snb.2017.11.035

[7] A.T. Güntner et al., Noninvasive Body Fat Burn Monitoring from Exhaled Acetone with Si-doped $\mathrm{WO}_{3}$-sensing Nanoparticles, Anal. Chem., 89 (2017) 10578-10584. doi: 10.1021/acs.analchem.7b02843 